\title{
Coleopterofauna em floresta e pastagem no norte do Paraná, Brasil, coletada com armadilha de solo
}

\author{
Ísis Meri Medri ${ }^{1}$ \\ José Lopes ${ }^{2}$
}

\begin{abstract}
Forest and pasture coleopterofauna in North of Paraná, Brazil, collected using pitfull traps. The environmental transformations constitute the principal cause of changes in biodiversity. This make the comparative studies between natural and altered environments very important. This research compare the beetle fauna at the following areas: isolated forest fragment and pasture at the secondary stage of succession. Beetles were collected using four pitfall traps baited with cattle meat in each area, fortnightly from November 1998 to April 1999. A total of 17,721 individuals from 30 families of Coleoptera in forest and 6,597 individuals belanging to 32 families in pasture was collected. The Sörensen index was used to calculate similarity, and Shannon index to calculate diversity. The qualitative similarity found was 0.70 and the quantitative similarity was 0.48 . The mean Shannon diversity index found was 1.90 for forest and 2.01 for pasture; these values were not significantly different by $\mathrm{T}$ test. It was concluded that the beetle decomposer fauna collected was representative in both environments, which also contributes in the decomposition process of organic matter, in soil fertilization and also in controlling egg and larvae populations of parasitic flies. KEY WORDS. Coleoptera, necrophagous, pitfall traps, forest fragment, pasture, decomposition
\end{abstract}

O estado do Paraná sofreu uma grande devastação de sua cobertura vegetal nativa e, atualmente, apresenta apenas alguns fragmentos remanescentes de florestas, circundadas por terras cultivadas ou por áreas de pastagem.

Os besouros apresentam vários hábitos alimentares, sendo que os especialistas podem ser exclusivamente fitófagos, predadores, parasitas, coprófagos ou necrófagos, e os generalistas associam estes hábitos, como por exemplo, os copronecrófagos.

Muitos coleópteros apresentam grande importância para o homem, agindo como polinizadores, controladores biológicos de artrópodes e plantas daninhas e decompositores de matéria orgânica. E muitos outros, causam danos ao alimentarem-se de produtos vegetais ou animais armazenados, sendo que a maioria das espécies de besouros não apresenta importância econômica direta, mas sua contribuição no funcionamento equilibrado do ecossistema não pode ser subestimada.

1) Curso de Graduação em Ciências Biológicas, Laboratório de Entomologia, Departamento de Biologia Animal e Vegetal, Universidade Estadual de Londrina. Caixa Postal 6001, Campus Universitário, 86051-990 Londrina, Paraná, Brasil.

2) Departamento de Biologia Animal e Vegetal, Universidade Estadual de Londrina. Caixa Postal 6001, Campus Universitário, 86051-990 Londrina, Paraná, Brasil. E-mail: jea@uel.br 
Os trabalhos de levantamento faunístico são necessários para a compreensão do funcionamento das comunidades e ecossistemas, e considerando a relevante importância de estudos comparativos entre ambientes naturais e antrópicos, este trabalho teve como objetivos: analisar e comparar a nível de família, a coleopterofauna atraída à armadilha de solo com isca de carne na floresta primária do Parque Estadual Mata dos Godoy e numa área de pastagem próxima a esta mata, e incrementar a Coleção Entomológica do Museu de Zoologia, Universidade Estadual de Londrina (MZUEL).

\section{MATERIAL E MÉTODOS}

O trabalho foi desenvolvido em área preservada do Parque Estadual Mata dos Godoy em Londrina, Paraná ( $\left.23^{\circ} 27^{\prime} \mathrm{S}, 51^{\circ} 15^{\prime} \mathrm{W}\right)$, com altitude em torno de 600 m e uma área de 680 ha, cuja vegetação é classificada fitogeograficamente como Floresta Estacional Semidecidual (BIANCHINI et al. no prelo).

Também foram feitas amostragens numa área de pastagem situada a aproximadamente $900 \mathrm{~m}$ do limite sul do Parque, a qual se diferencia das pastagens tradicionais por apresentar muitas ervas e arbustos, além de algumas árvores distribuídas aleatoriamente pela área, pelo que se nota já estar ocorrendo o início do processo de sucessão secundária, mesmo sendo uma área cercada, mas com pouco pisoteamento pelo gado.

O clima da região, conforme MAACK (1981) é, segundo a classificação de Köeppen, do tipo Cfa, ou seja, clima subtropical úmido, com verões quentes e invernos com geadas pouco freqüentes e sem estação seca definida. A média pluviométrica anual é de $1.714,7 \mathrm{~mm}$ e a temperatura média anual é de $21^{\circ} \mathrm{C}$.

O solo da região é predominantemente argiloso e profundo, com boa porosidade e drenagem e apresenta cor arroxeada, sendo classificado como Terra Roxa Estruturada Eutrófica e a área amostrada do Parque Estadual Mata dos Godoy, apresenta solo do tipo Latossolo Roxo Eutrófico (EMBRAPA 1984).

O Parque Estadual Mata dos Godoy apresenta uma fauna muito representativa para a região, abrigando inclusive mamíferos de grande porte. Com relação à mastofauna da área de pastagem adjacente ao parque, apesar de comportar algumas espécies de roedores, é composta predominantemente por bovinos e eqüinos.

Em ambos os locais de estudo, área de pastagem e floresta primária, foram instaladas quatro armadilhas de solo que se constituíram em recipientes plásticos de $23 \mathrm{~cm}$ de comprimento e de $9,5 \mathrm{~cm}$ de diâmetro, com abertura ao nível do solo e com distanciamento de $20 \mathrm{~m}$ entre elas. No bordo superior interno de cada recipiente foi acoplado, através de fio de arame, um pequeno frasco com tampa perfurada, servindo de porta isca (Fig. 1). Em cada frasco porta isca, foram colocadas $50 \mathrm{~g}$ de carne bovina moída que, ao se decompor, serve como atrativo para os coleópteros necrófagos. Ao redor da abertura de cada armadilha foi colocado um plástico de $45 \times 46,5 \mathrm{~cm}$, para evitar a queda de terra no interior da armadilha, o que dificultaria a triagem do material biológico coletado.

Em cada área do experimento, uma das armadilhas permaneceu sem isca servindo como testemunha. Todas as armadilhas receberam $200 \mathrm{ml}$ de solução formol 4\% como líquido fixador, conforme indicado por LOPES et al. (1994). 


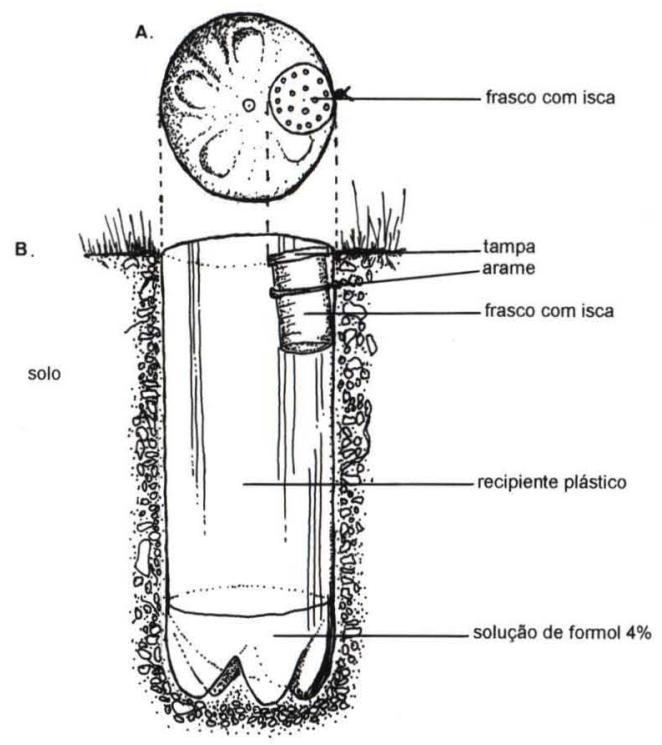

Fig. 1. Armadilha de solo. (A) frasco porta isca, (B) recipiente plástico e (C) solução formol $4 \%$.

As coletas tiveram periodicidade quinzenal, durante o período de 24 de novembro de 1998 a 27 de abril de 1999. A cada coleta, o conteúdo das armadilhas foi transferido para frascos de transporte devidamente identificados.

No laboratório, o material resultante das coletas foi colocado numa bandeja, separado e acondicionado em frascos contendo álcool $70 \%$. As famílias de macrocoleópteros foram identificadas com o uso da Chave para as famílias de Coleoptera de BORROR \& DELONG (1969). Os microcoleópteros foram separados, conforme às variações morfológicas e alguns representantes de cada tipo foram posteriormente enviados a especialista para identificação.

Para verificar se houve ou não atratividade da isca utilizada sobre os coleópteros, foi aplicado o teste de Friedman (LOPES et al. 1994). Com a finalidade de avaliar a diversidade das famílias coletadas na Mata dos Godoy e na área de pastagem adjacente, foi utilizado o índice de Shannon (MAGURRAN 1988) determinado pela fórmula $H^{\prime}=-\Sigma$ pi.Lnpi. E para comparar a existência ou não de diferença significativa entre os valores de diversidade obtidos nos locais amostrados, foi utilizado o teste $\mathrm{T}$.

Foram feitas comparações qualitativas baseadas na presença e na ausência das famílias de Coleoptera dos locais amostrados mediante o índice de similaridade qualitativa de Sörensen (MAgurRAn 1988), através da fórmula $\mathrm{Cs}=2 \mathrm{j} /(\mathrm{a}+\mathrm{b})$. O índice de Sörensen de similaridade quantitativa das famílias de Coleoptera dos ambientes de pasto e mata, foi estimado mediante a fórmula $\mathrm{Cn}=2 \mathrm{jN} /(\mathrm{aN}+\mathrm{bN})$. O índice de similaridade de Sörensen varia de 0 a 1 , sendo que o valor 1 representa o máximo de semelhança entre os locais amostrados.

Todo o material coletado foi depositado no MZUEL. 


\section{RESULTADOS E DISCUSSÃO}

As doze coletas realizadas, entre o período de novembro de 1998 a abril de 1999, totalizaram 24.318 coleópteros, sendo 17.721 (73\%) provenientes da área de floresta e 6.597 (27\%) da área de pastagem.

A tabela I agrupa os resultados da distribuição dos coleópteros obtidos nas armadilhas controle e nas que receberam isca. Durante todo o experimento, duas armadilhas foram encontradas desenterradas, provavelmente por coatis, impossibilitando a obtenção de coleópteros nestas ocasiões. Pela análise destes resultados, através do teste de Friedman, verificou-se diferença significativa $(\mathrm{P}<0,05)$ entre $o$ total coletado nas armadilhas controle e nas com isca, sendo que entre estas últimas não houve diferença comprovando assim que a isca utilizada foi atrativa para muitos coleópteros.

Tabela I. Número de exemplares de Coleoptera coletados entre novembro/1998 a abril/1999, em armadilhas controles (P1 e M1) e iscadas com carne (P2, P3, P4, M2, M3 e M4) instaladas em área de floresta (Mata dos Godoy) $(M)$ e em área de pastagem $(P)$.

\begin{tabular}{crrrrrrrr}
\hline \multicolumn{1}{c}{ Datas } & P1 & \multicolumn{1}{c}{$\mathrm{P} 2$} & \multicolumn{1}{c}{$\mathrm{P} 3$} & \multicolumn{1}{c}{$\mathrm{P} 4$} & $\mathrm{M} 1$ & $\mathrm{M} 2$ & $\mathrm{M} 3$ & $\mathrm{M} 4$ \\
\hline $24 / 11 / 1998$ & 65 & 102 & 271 & 145 & 77 & 310 & 251 & 267 \\
$11 / 12 / 1998$ & 41 & 857 & 166 & 100 & 213 & 683 & 1890 & 737 \\
$22 / 12 / 1998$ & 3 & 402 & 325 & 249 & 57 & 605 & 1233 & 1032 \\
$05 / 01 / 1999$ & 44 & 132 & 191 & 183 & 12 & 360 & 770 & 406 \\
$19 / 01 / 1999$ & 37 & 46 & 204 & - & 21 & 272 & 426 & 157 \\
$02 / 02 / 1999$ & 16 & 104 & 84 & 33 & 10 & 274 & 386 & 266 \\
$17 / 02 / 1999$ & 26 & 156 & 145 & 159 & 36 & 613 & 501 & 327 \\
$02 / 03 / 1999$ & 6 & 220 & 149 & 241 & 24 & 214 & 402 & 267 \\
$16 / 03 / 1999$ & 11 & 131 & 118 & 97 & 42 & 201 & 606 & 378 \\
$30 / 03 / 1999$ & 6 & 384 & 202 & 334 & 46 & 345 & 384 & 444 \\
$13 / 04 / 1999$ & 17 & 90 & 121 & 91 & 56 & 277 & 394 & 399 \\
$27 / 04 / 1999$ & 6 & - & 55 & 32 & 9 & 385 & 415 & 241 \\
\hline Total & 278 & 2624 & 2031 & 1664 & 603 & 4539 & 7658 & 4921 \\
\hline
\end{tabular}

(-) armadilha desenterrada (interferência de animais).

A distribuição dos espécimens representantes das 40 famílias de Coleoptera coletadas, bem como os índices de diversidade de Shannon e de similaridade de Sörensen, são demonstrados na tabela II.

Os resultados (Tab. II), indicam presença de 22 famílias ocorrendo em ambos os ambientes de coleta, e 10 delas foram exclusivas da área de pastagem, enquanto que 8 ocorreram somente na floresta. No ambiente de floresta amostrado foi obtido um número maior de besouros do que na área de pastagem, o que pode ser devido às condições microclimáticas favoráveis e, provavelmente, a maior oferta de recursos alimentares.

A isca foi um atrativo para os coleópteros das famílias Histeridae, Hybosoridae, Leiodidae, Nitidulidae, Ptiliidae, Scarabaeidae e Staphylinidae (Tab. III). Destas, as famílias Histeridae, Staphylinidae (alguns), além das famílias Carabidae, Cicindelidae, Coccinellidae, Elateridae (alguns), Hydrophilidae e Scydmaenidae podem ser encontradas em matéria animal ou vegetal em decomposição, mas são predadoras de outros insetos que vivem nestes materiais. Desta forma, a isca pode ter papel intermediário na aproximação destes insetos predadores. 
Tabela II. Familias de Coleoptera coletadas em armadilhas de solo com isca de carne, em ambientes de floresta primária e de pastagem adjacente no municipio de Londrina, Paraná, entre novembro de 1998 a abril de 1999, indicando o nível trófico, índice de Shannon e as similaridade quantitativa e qualitativa.

\begin{tabular}{|c|c|c|c|c|c|c|}
\hline Familias & Grupo trófico & Área de pastagem & Porcentagem & Mata dos Godoy & Porcentagem & Total \\
\hline Anobiidae & $x$ & 0 & 0,00 & 1 & $<0,01$ & 1 \\
\hline Anthicidae & $\mathrm{S}$ & 46 & 0,69 & 0 & 0,00 & 46 \\
\hline Biphyllidae & $\mathrm{F}$ & 0 & 0,00 & 1 & $<0,01$ & 1 \\
\hline Buprestidae & $\mathrm{H}$ & 1 & 0,01 & 0 & 0,00 & 1 \\
\hline Carabidae & $\mathrm{Pr}$ & 5 & 0,08 & 8 & 0,04 & 13 \\
\hline Cerambycidae & $\mathrm{H}$ & 0 & 0,00 & 2 & 0,01 & 2 \\
\hline Chelonariidae & $\mathrm{H}$ & 1 & 0,01 & 0 & 0,00 & 1 \\
\hline Chrysomelidae & $\mathrm{H}$ & 328 & 4,97 & 7 & $<0,01$ & 335 \\
\hline Cicindelidae & $\mathrm{Pr}$ & 1 & 0,01 & 0 & 0,00 & 1 \\
\hline Coccinellidae & $\mathrm{Pr}$ & 0 & 0,00 & 1 & $<0,01$ & 1 \\
\hline Colydiidae & $\mathrm{F}$ & 1 & 0,01 & 0 & 0,00 & 1 \\
\hline Corylophidae & $\mathrm{F}$ & 26 & 0,39 & 1 & $<0,01$ & 27 \\
\hline Curculionidae & $X, F, X F$ & 2 & 0,03 & 128 & 0,72 & 130 \\
\hline Dermestidae & $\mathrm{S}$ & 2 & 0,03 & 0 & 0,00 & 2 \\
\hline Elateridae & $\mathrm{H}, \mathrm{Pr}, \mathrm{X}$ & 2 & 0,03 & 4 & 0,02 & 6 \\
\hline Erotylidae & $\mathrm{F}$ & 0 & 0,00 & 2 & 0,01 & 2 \\
\hline Histeridae & $\mathrm{Pr}$ & 531 & 8,05 & 507 & 2,86 & 1038 \\
\hline Hybosoridae & $S$ & 185 & 2,80 & 2817 & 15,89 & 3002 \\
\hline Hydrophilidae & $\operatorname{Pr}$ & 105 & 1,59 & 232 & 1,31 & 337 \\
\hline Laemophloeidae & $\mathrm{F}$ & 2 & 0,03 & 0 & 0,00 & 2 \\
\hline Lagriidae & $\mathrm{H}, \mathrm{S}$ & 3 & 0,05 & 0 & 0,00 & 3 \\
\hline Lathridiidae & $\mathrm{F}$ & 0 & 0,00 & 1 & $<0,01$ & 1 \\
\hline Leiodidae & F, S & 107 & 1,62 & 1704 & 9,61 & 1811 \\
\hline Melandryidae & $\mathrm{F}$ & 0 & 0,00 & 1 & $<0,01$ & 1 \\
\hline Mordellidae & $\mathrm{H}$ & 1 & 0,01 & 1 & $<0,01$ & 2 \\
\hline Mycetophagidae & $\mathrm{F}$ & 1 & 0,01 & 0 & 0,00 & 1 \\
\hline Nitidulidae & $\mathrm{F}, \mathrm{H}, \mathrm{S}$ & 881 & 13,35 & 596 & 3,36 & 1477 \\
\hline Phalacridae & $\mathrm{F}, \mathrm{H}$ & 4 & 0,06 & 0 & 0,00 & 4 \\
\hline Platypodidae & $\mathrm{F}, \mathrm{H}$ & 0 & 0,00 & 1 & $<0,01$ & 1 \\
\hline Pselaphidae & $\mathrm{H}$ & 2 & 0,03 & 3 & 0,02 & 5 \\
\hline Ptiliidae & F, S & 1202 & 18,22 & 4412 & 24,89 & 5614 \\
\hline Ptilodactylidae & S & 2 & 0,03 & 9 & 0,05 & 11 \\
\hline Scarabaeidae & $\mathrm{H}, \mathrm{S}$ & 956 & 14,49 & 3113 & 17,56 & 4069 \\
\hline Scolytidae & $\mathrm{H}$ & 140 & 2,12 & 294 & 1,66 & 434 \\
\hline Scydmaenidae & $\mathrm{Pr}$ & 2 & 0.03 & 4 & 0,02 & 6 \\
\hline Silphidae & $\mathrm{S}$ & 4 & 0.06 & 1 & $<0,01$ & 5 \\
\hline Silvanidae & $\mathrm{F}$ & 26 & 0,39 & 4 & 0,02 & 30 \\
\hline Staphylinidae & F, Pr, S & 2023 & 30,66 & 3860 & 21,78 & 5883 \\
\hline Tenebrionidae & F, S & 4 & 0.06 & 1 & $<0,01$ & 5 \\
\hline Trogidae & s & 1 & 0,01 & 5 & 0,03 & 6 \\
\hline Total & & 6597 & 100,00 & 17721 & 100,00 & 24318 \\
\hline Total de familias & & 32 & & 30 & & 40 \\
\hline$H^{\prime}$ & & 2,01 & & 1,90 & & \\
\hline Cs & & & & & & 0,70 \\
\hline $\mathrm{Cn}$ & & & & & & 0,48 \\
\hline
\end{tabular}

( $\left.\mathrm{H}^{\prime}\right)$ Índice de Shannon, (Cs) similaridade qualitativa, ( $\mathrm{Cn}$ ) similaridade quantitativa (Sörensen), (F) fungivoro, $(\mathrm{H})$ herbivoro, $(\mathrm{Pr})$ predador, $(\mathrm{S})$ saprófago, $(\mathrm{X})$ xilófago, $(\mathrm{XF})$ xilomicetófago (Fonte: Hammond 1990 apud DIHAM et al. 1998).

Nos resultados nota-se, que as famílias de hábito alimentar exclusivamente saprófago ou de hábito alimentar misto, foram as mais abundantes: Hybosoridae, Leiodidae, Nitidulidae, Ptiliidae, Scarabaeidae e Staphylinidae; com exceção de Histeridae cujos representantes são exclusivamente predadores, mostrando a atratividade da isca (Tab. III). 
Analisando somente as armadilhas iscadas, constatou-se que a maioria dos crisomelídeos $(73,2 \%)$ foram capturados na armadilha 3 da pastagem. Por não haver uma distribuição homogênea entre as armadilhas, conjectura-se que a queda dos exemplares foi acidental, podendo estes besouros terem sido atraídos por alguma planta próxima à armadilha, já que estes são exclusivamente herbívoros. A mesma situação foi observada para os curculionídeos, onde $72,4 \%$ dos exemplares pertencentes a esta família foram apanhados na armadilha 3 da floresta. Para os escolitídeos, houve uma distribuição uniforme entre as armadilhas iscadas, excetuando a armadilha 3 da floresta onde a densidade foi maior (Tab. III).

Tabela III. Número médio do total para as famílias de Coleoptera mais abundantes coletadas em armadilha de solo, controle e com isca de carne, instalada em ambiente de floresta primária e de pastagem adjacente no município de Londrina, Paraná, entre novembro de 1988 e abril de 1999, mostrando a atração da isca e o nivel trófico para cada familia.

\begin{tabular}{lrrl}
\hline \multicolumn{1}{c}{ Familias } & Controle & Iscada & \multicolumn{1}{c}{ Grupo trófico } \\
\hline Crysomelidae & 20,0 & 49,2 & $\mathrm{H}$ \\
Curculionidae & 7,0 & 19,3 & $\mathrm{H}, \mathrm{X}, \mathrm{XF}$ \\
Histeridae & 1,5 & 172,5 & $\mathrm{Pr}$ \\
Hydrophilidae & 11,0 & 52,3 & $\mathrm{Pr}$ \\
Hybosoridae & 43,0 & 486,0 & $\mathrm{~S}$ \\
Leiodidae & 1,0 & 301,5 & $\mathrm{~F}, \mathrm{~S}$ \\
Nitidulidae & 139,5 & 199,7 & $\mathrm{~F}, \mathrm{H}, \mathrm{S}$ \\
Ptilidae & 77,5 & 910,0 & $\mathrm{~F}, \mathrm{~S}$ \\
Scarabaeidae & 17,0 & 660,0 & $\mathrm{H}, \mathrm{S}$ \\
Scolytidae & 41,0 & 58,6 & $\mathrm{H}$ \\
Staphylinidae & 62,5 & 959,7 & $\mathrm{~F}, \mathrm{Pr}, \mathrm{S}$ \\
\hline
\end{tabular}

(F) Fungivoro, (H) herbívoro, $(\mathrm{Pr})$ predador, (S) saprófago, (X) xilófago, (XF) xilomicetófago (Fonte: HAMMOND 1990 apud DiHAm et al. 1998).

As famílias mais abundantes na floresta foram: Ptiliidae (25\%), Staphylinidae $(22 \%)$, Scarabaeidae (18\%), Hybosoridae (16\%) e Leiodidae $(10 \%)$, as quais resultaram em $91 \%$ do total coletado neste ambiente, enquanto que, na área de pastagem, predominaram: Staphylinidae (31\%), Ptiliidae (18\%), Scarabaeidae $(15 \%)$, Nitidulidae (13\%) e Histeridae $(8 \%)$, com $85 \%$ do total obtido neste local (Fig. 2). Estas famílias, mesmo presentes em alta densidade na floresta, mostraramse adaptadas ao ambiente antrópico.

Em ambos os ambientes de coleta a maioria das famílias obtidas foi representada por poucos indivíduos (Fig. 3). A teoria de HALFFTER (1991) diz que a distribuição em que poucas espécies são muito abundantes e muitas espécies são representadas por um número restrito de indivíduos, é característica de florestas tropicais. Embora os resultados obtidos na atual pesquisa mostre esta tendência, o fato de existir a atração pela isca, mascara a constatação de fenômeno desta natureza.

O valor de diversidade, índice de Shannon, foi igual a 1,90 para a floresta e 2,01 para a área de pastagem, não apresentando através do teste $\mathrm{T}(\mathrm{P}>0,05 \mathrm{e}<$ $0,10)$, diferença significativa na diversidade de famílias de Coleoptera para ambos os ambientes.

Obteve-se, pelo índice de Sörensen, um valor maior de similaridade qualitativa $(0,70)$ entre os dois ambientes de coleta do que de similaridade quantitativa 

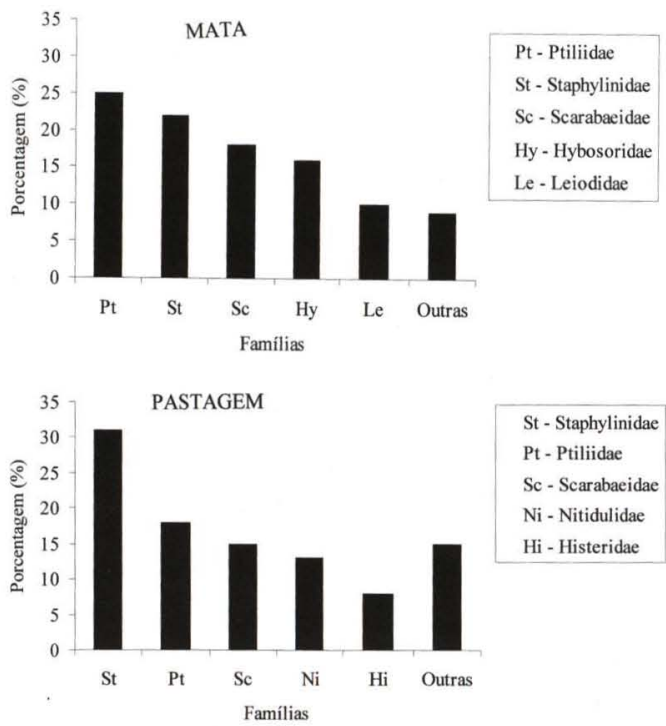

Fig. 2. Porcentagem das familias de Coleoptera mais abundantes coletadas em armadilha de solo com isca de carne, no Parque Estadual Mata dos Godoy e em área de pastagem próxima, entre novembro de 1998 a abril de 1999.
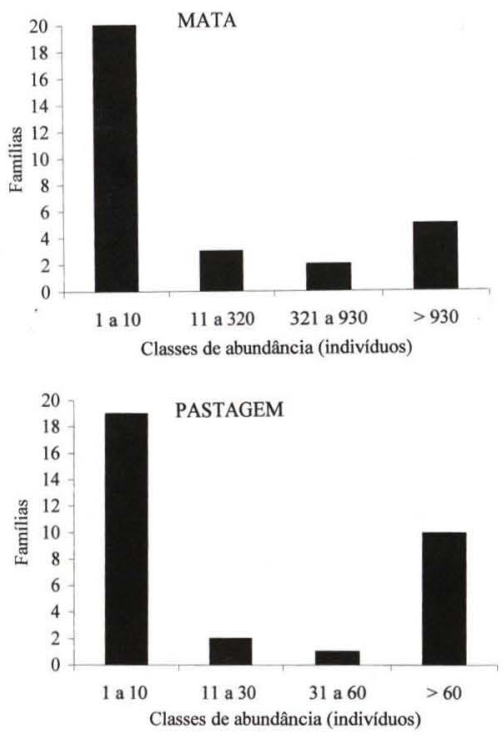

Fig. 3. Número das famílias de Coleoptera relacionado ao número de indivíduos coletados na Mata dos Godoy em armadilha de solo com isca de carne e em área de pastagem próxima, entre novembro de 1998 a abril de 1999.

Revta bras. Zool. 18 (Supl. 1): 125 - 133. 2001 
$(0,48)$, indicando que ao nível de família, a fauna de coleópteros dos locais analisados difere mais no número de indivíduos do que na composição, ou seja, a mesma família pode ocorrer em ambos os locais mas apresentando uma abundância maior em um dos ambientes.

A fragmentação dos habitats naturais pode induzir mudanças na abundância e na riqueza de coleópteros decompositores (KLEIN 1989), o que pode causar impactos no suprimento e retenção de nutrientes no solo (NEALIS 1977), na dispersão de sementes (EsTRADA \& CoATES-EsTRADA 1991), no controle de moscas parasitas de vertebrados (BERGSTROM et al. 1976) e, em conseqüência disso, pode afetar a estabilidade do ecossistema, já que muitas espécies dasta família de inseto podem estar envolvidas nestes processos.

\section{CONCLUSÕES}

A carne bovina utilizada no experimento mostrou-se atrativa para muitos coleópteros, principalmente para as famílias Histeridae, Hybosoridae, Leiodidae, Nitidulidae, Ptiliidae, Scarabaeidae e Staphylinidae.

A maioria das famílias de Coleoptera coletada nas armadilhas de solo instaladas no Parque Estadual Mata dos Godoy e na área de pastagem próxima, foi representada por poucos indivíduos, enquanto apenas algumas como Staphylinidae, Ptiliidae, Scarabaeidae e Hybosoridae foram muito abundantes.

Obteve-se um número quase três vezes maior de besouros na Mata dos Godoy comparado ao total obtido na área de pastagem, o que pode ser devido às condições microclimáticas favoráveis e à disponibilidade de recursos alimentares que a floresta primária oferece, tais como excrementos e carcaças da fauna silvestre e matéria vegetal em decomposição.

De maneira geral, constatou-se, através do índice de Sörensen, maior similaridade qualitativa do que quantitativa, indicando que ocorre maior diferença entre o pasto e a mata, no número de coleópteros do que na composição destes, ou seja, ocorre mudança das famílias dominantes em cada ambiente.

Em relação à diversidade, os valores do índice de Shannon resultantes demonstraram pelo teste $\mathrm{T}$, que não houve diferença significativa na composição faunística em relação as famílias de Coleoptera registradas para os ambientes analisados.

Concluiu-se que a coleopterofauna obtida em armadilha de solo iscada com carne, foi representativa nos dois ambientes. Para os coleópteros pertencentes as famílias mais abundantes, pode se inferir que foram atraídos pela isca, o que indica hábito necrófago, podendo assim estar contribuindo no processo de decomposição da matéria orgânica, na fertilização do solo e no controle de larvas de moscas, os quais constituem importantes processos para o equilíbrio do ecossistema.

AGRADECIMENTOS. Ao Sr. Ayr de Moura Bello pela identificação da amostra das famílias de microcoleópteros obtidos; a José Marcelo Torezan pelo auxílio nos cálculos dos índices ecológicos e valiosas sugestões; a Waldir Medri, José Carlos Dalmas e José da Costa Soeiro pelos cálculos estatísticos; a Cláudio Müller pela revisão do artigo e ao $\mathrm{CNPq}$ pela concessão de Bolsa de Iniciação Científica. 


\section{REFERÊNCIAS BIBLIOGRÁFICAS}

Bergstrom, B.C.; R.L. Maki \& B.A. Werner. 1976. Small dung beetles as biological control agent: laboratory studies of beetle action on trichostongylid eggs in sheep and cattle feces. Proc. Helminthol. Soc. Wash. 43: 171-174.

Bianchini, E.; J.A. Pimenta \& F.A.M. Santos. (no prelo). Spatial and temporal variation in the canopy cover in a tropical semi-deciduous forest. Brazil. Arch. Biol. Technol., Curitiba, 44 (1).

Borror, D.J. \& D.M. DeLonG. 1969. Introdução ao estudo dos insetos. São Paulo, Edgard-Blücher Ltda, $1^{a}$ ed., 653p.

Diham, R.K.; P.M. Hammond; J.H. Lawton; P. EgGleton \& N.E. StoRK. 1998. Beetle species responses to tropical forest fragmentation. Ecol. Monogr. 68 (3): 295-323.

EMBraPA (Empresa Brasileira de Pesquisa Agropecuária). 1984. Levantamento de reconhecimento de solos do Estado do Paraná. Curitiba, Embrapa, 791p.

Estrada, A. \& R. Coates-Estrada. 1991. Howling monkeys (Alouatta palliata) dung beetles (Scarabaeidae) and seed dispersal: ecological interactions in the tropical rain florest of Los Tuxtlas, Veracruz, Mexico. Jour. Trop. Ecol. 7: 459-474.

HALfFter, G. 1991. Historical and ecological factors determining the geographical distribuition of beetles (Coleoptera: Scarabaeidae: Scarabaeinae). Folia Entomol. Mexicana 82: 195-238.

KLEIN, B.C. 1989. Effects of forest fragmentation on dung and carrion beetle cpmmunities in central Amazonia. Ecology 6: 1715-1725.

LOPES, J.; I. CONCHON; S.K. YuZAWA \& R.R.C. KuHnLEIN. 1994. Entomofauna do Parque Estadual Mata dos Godoy: II. Scarabaeidae (Coleoptera) coletados em armadilhas de solo. Semina, Londrina, 15 (2): 121-127.

MaACK, R. 1981. Geografia Física do Estado do Paraná. Rio de Janeiro, J. Olympio, $2^{\mathrm{a}}$ ed., 450p. Magurran, A.E. 1988. Ecological Diversity and Its Measurement. New Jersey, Princeton, 179p.

NEALIS, V.G. 1977. Habitat association an community analysis of south Texas dung beetles (Coleoptera: Scarabaeidae). Canad. Jour. Zool. 55: 138-147.

Recebido em 11.V.2000; aceito em 20.VI.2001 